\title{
Bacterial Population Control with Macroscopic HKUST Crystals
}

\author{
Rafael Ballesteros-Garrido, ${ }^{\mathrm{a}, \mathrm{b}, *}$, Roser Montagud-Martínez ${ }^{\mathrm{a}, \mathrm{b}}$, and Guillermo Rodrigo ${ }^{\mathrm{a}, *}$ \\ a Instituto de Biología Integrativa de Sistemas (I2SysBio), CSIC - U. Valencia, 9 Cat. Agustin Escardino, 46980 \\ Paterna, Spain \\ Supporting Information (SI) available.
}

\begin{abstract}
Macroscopic HKUST crystals were shown to release significant amounts of copper in saline medium at a slow rate, which was exploited to control the growth of a bacterial population. This was achieved in both liquid and solid media, the latter illustrating the local effect of the crystals. In addition, these nanostructured crystals of observable size were loaded with chloramphenicol to exert a joint metal-antibiotic action, going beyond the traditional oligodynamic effect.
\end{abstract}

Keywords: antibiotics, controlled release, metal-organic framework, nanobiotechnology, size.

\section{Introduction}

Bacterial infections represent one of the main causes of death at a global scale, especially in children and in lowincome countries ${ }^{1}$. Thus, the ability to control bacterial populations is an important challenge that today can involve different disciplines; for example, to warrant a biosafe food and water supply or to avoid the spread within the body once an infection focus has appeared. In a modern era in which the continuous emergence of antibiotic resistances is a threat ${ }^{2}$ and in which the progress of effective treatments (in biomedicine and beyond) demands increasing precision ${ }^{3}$, novel repression ways are certainly needed to fight bacteria.

Consequently, the possibility of using a suitable nanostructured material to inhibit the growth of a given bacterial population is appealing ${ }^{4}$. Indeed, self-assembled nanostructures have emerged in recent years as new efficient and programmable elements for the distribution of active biochemical compounds (drugs) in different biological contexts 5,6 , going beyond the traditional applications of catalysis and gas storage ${ }^{7}$. In part, this has allowed addressing issues associated with efficacy, toxicity, and stability of such drugs. Moreover, some nanostructures have been shown to have antibacterial activity, especially those involving metals ${ }^{8,9}$. However, most of the developments are focused on the nanoscale (particles of $\mathrm{nm}$ ). This may limit the applicability of the technology because those materials are difficult to control once disseminated ${ }^{10}$.

In this work, by contrast, we decided to investigate the use of nanostructured materials of macroscopic size (particles of $\mathrm{mm}$ ) to fight bacteria. The application of such materials may offer new nanobiotechnological horizons ${ }^{11}$, but they remain largely unexplored. Potential advantages are i) a slow release kinetics thanks to a smaller surface per volume ratio, following the application of the Fick's laws to such systems ${ }^{12}$; ii) the ability to interact with the cells of the medium and experience phenomena such as gravity and orientation; or iii) the ability to manually locate materials in a controlled way to then remove them (and even reuse them with new loads $)^{13}$.

Among the different materials, metal-organic frameworks (MOFs) ${ }^{14}$ have achieved great relevance in biomedicine over the last years ${ }^{6}$. In particular, we here focused on HKUST-1 $\left(\mathrm{Cu}_{3} \mathrm{BTC}_{2}\right)^{15}$, or simply HKUST, aimed at controlling a population of Escherichia coli cells in lab conditions (Fig. 1). We chose this material because it can be prepared to create mm-sized crystals (see Fig. $\mathrm{S} 1$ in $\mathrm{SI})^{16}$, and we considered $E$. coli as a model of gram-negative bacteria (note that many pathogenic bacteria are of this type ${ }^{17}$ ). Copper, the metal in HKUST1 , is well-known to have antimicrobial activity (what is called oligodynamic effect) ${ }^{18}$. MOFs have been reported to experience water and moisture sensitivity ${ }^{19,20}$, but to what extent can this be profitable? In the following, we present results that show that HKUST-1 crystals of observable size are suitable elements for controlled copper release to the medium (in the form of $\mathrm{Cu}^{2+}$ ), at the same time that they can serve as containers for other deliverable molecules (e.g., antibiotics), which effectively leads to bacterial repression.

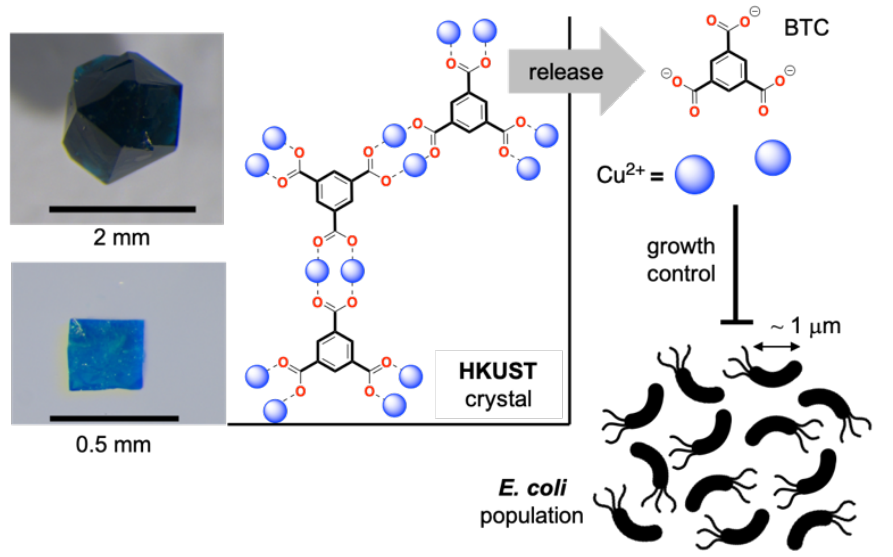

Figure 1: Macroscopic HKUST crystals can control the growth of a bacterial population (e.g., of $E$. coli). Copper release (in the form of $\mathrm{Cu}^{2+}$ ) due to partial crystal degradation is instrumental. Depending on the reaction conditions, the size of the observable crystal can greatly vary (from 0.2 to $2 \mathrm{~mm}$ edge).

\section{Results}

We observed that macroscopic HKUST-1 crystals release copper in saline medium (in particular, LB medium), but not in water (Fig. 2a); arguably, due to electrostatic forces during the competition between lattice energy, solvation, and coordination with nitrogen-based compounds. This was clearly observed by a bluish coloration of the LB medium. The kinetics of the copper release was well explained by an exponential decay model in increasing form. That is, $x(t)=x_{\max }\left(1-e^{-k t}\right)$, where $x(t)$ represents the amount of $\mathrm{Cu}^{2+}$ in the medium at time $t, x_{\max }$ the maximal amount of this metal observed in the medium, and $k$ the release rate. This corresponds to the differential 
equation $\mathrm{d} x / \mathrm{d} t=k\left(x_{\max }-x\right)$. At initial times, the release flux is roughly constant $\left(k \cdot x_{\max }\right)$. But, as the amount of $\mathrm{Cu}^{2+}$ in the medium increases, the flux is lower (as the electrostatic forces equilibrate). The absorbance at 650 $\mathrm{nm}$ was a good proxy to monitor the release (see Figs. S2, S3 in SI). Due to the direct correlation between absorbance and $\mathrm{Cu}^{2+}$ concentration, obtained with control solutions, in Fig. S4 in SI we present the kinetics of the release in terms of concentration. Despite immersion in water was already shown to induce structural changes that can be observed by powder X-ray diffraction (PXRD), scanning electron microscopy, and even visually ${ }^{20}$, we show here that copper cations are not released in this pure medium.
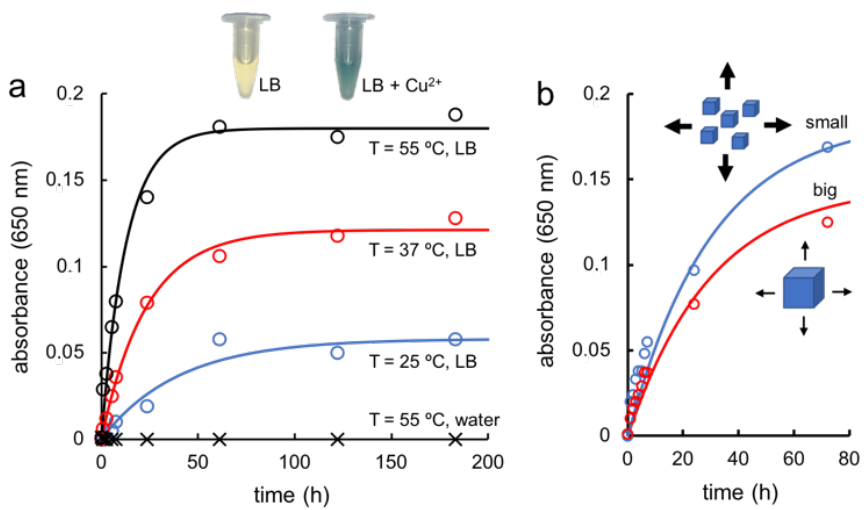

Figure 2: Copper release from macroscopic HKUST crystals. a) Release kinetics in LB medium (or water) at different temperatures $(25,37$, and 55 $\left.{ }^{\circ} \mathrm{C}\right)$. b) Release kinetics from big $(1-2 \mathrm{~mm})$ or small $(\sim 0.2 \mathrm{~mm})$ crystals at $37^{\circ} \mathrm{C}$. Circles, experimental data; lines, theoretical models.

At $37{ }^{\circ} \mathrm{C}$, we fitted $k=0.0428 \pm 0.0032 \mathrm{~h}^{-1}$ and $x_{\max }=$ $0.1213 \pm 0.0026\left(R^{2}=0.9942\right)$, for $10 \mathrm{mg} \mathrm{HKUST-1}$ (particles of $\sim 0.2 \mathrm{~mm}$ size) immersed in $2 \mathrm{~mL}$ LB. That is, the system takes $3 / k=70 \mathrm{~h}$ in reaching the equilibrium $\left(95 \%\right.$ of $\left.x_{\max }\right)$. In a colder environment $\left(25^{\circ} \mathrm{C}\right)$, the kinetics is slower (almost the half) and the maximal amount released lower, obtaining $k=0.0251 \pm 0.0006 \mathrm{~h}^{-1}$ and $x_{\max }=0.0581 \pm 0.0047\left(R^{2}=0.9549\right)$; whilst in a hotter environment $\left(55^{\circ} \mathrm{C}\right)$, the kinetics is faster (almost the double) and the maximal amount released higher, giving $k=0.0774 \pm 0.0077 \mathrm{~h}^{-1}$ and $x_{\max }=0.1801 \pm 0.0049$ $\left(R^{2}=0.9863\right)$. Qualitatively, this is in tune with a thermodynamic view of this process of partial crystal degradation.

Because of diffusion and degradation are surfacedriven phenomena, we hypothesized that the bigger the HKUST-1 crystal, the higher the ability and slower the kinetics of copper release. To prove this, we prepared big and small crystals of observable size (particles of 1-2 mm edge and $\sim 0.2 \mathrm{~mm}$ edge, respectively) to be assayed in the same conditions (maintaining constant the total material mass; see Fig. S5 in SI). Results shown in Fig. 2b indeed confirmed our hypothesis (with $k=0.0333 \pm$ $0.0048 \mathrm{~h}^{-1}$ and $x_{\max }=0.1472 \pm 0.0051, R^{2}=0.9734$ in the case of big material; and $k=0.0399 \pm 0.0054 \mathrm{~h}^{-1}$ and $x_{\max }$ $=0.1853 \pm 0.0060, R^{2}=0.9733$ for small material). We found statistically significant the difference between the two fittings, taking the mean and standard deviation of $k$ and $x_{\max }$ (Welch's $t$-tests, two-tailed $P<0.05$ in both cases). Moreover, we found that the copper release from a microscopic HKUST-1 material (grinding mm-sized particles) is substantially faster (see Fig. $\mathbf{S 6}$ in $\mathbf{S I}$ ).

Given the ability of HKUST-1 crystals to release copper in LB medium, and knowing the oligodynamic effect of this metal ${ }^{18}$, we wondered if a bacterial population could be maintained under control with our materials of observable size. To tackle this important question, we took diluted $E$. coli cultures (1:100 from overnight, strain $\mathrm{DH} 5 \alpha$ ) to then grow them in liquid medium (LB) with and without crystals (at $37^{\circ} \mathrm{C}$ with shaking). The absorbance at $500 \mathrm{~nm}$ was now a good proxy of bacterial amount, avoiding potential interfering signals from copper at $>550 \mathrm{~nm}$ (Fig. S3). As this metal acts as a bacteriostatic agent on $E$. coli, the use of absorbance is justified to monitor the repression. We found that these observable crystals, both big and small, inhibit the growth of $E$. coli (Fig. 3a). The differences reported (at 4 and $24 \mathrm{~h}$ after material addition, $10 \mathrm{mg}$ ) were indeed significant (Welch's $t$-tests, two-tailed $P<$ 0.05) compared to populations grown in absence of materials. However, the inhibition achieved with big crystals at $4 \mathrm{~h}$ was minimal. The different cultures almost did not grow from 4 to $24 \mathrm{~h}$ with material, pointing out that a threshold level in the $\mathrm{Cu}^{2+}$ amount in the medium needs to be reached to ensure growth control. Moreover, the
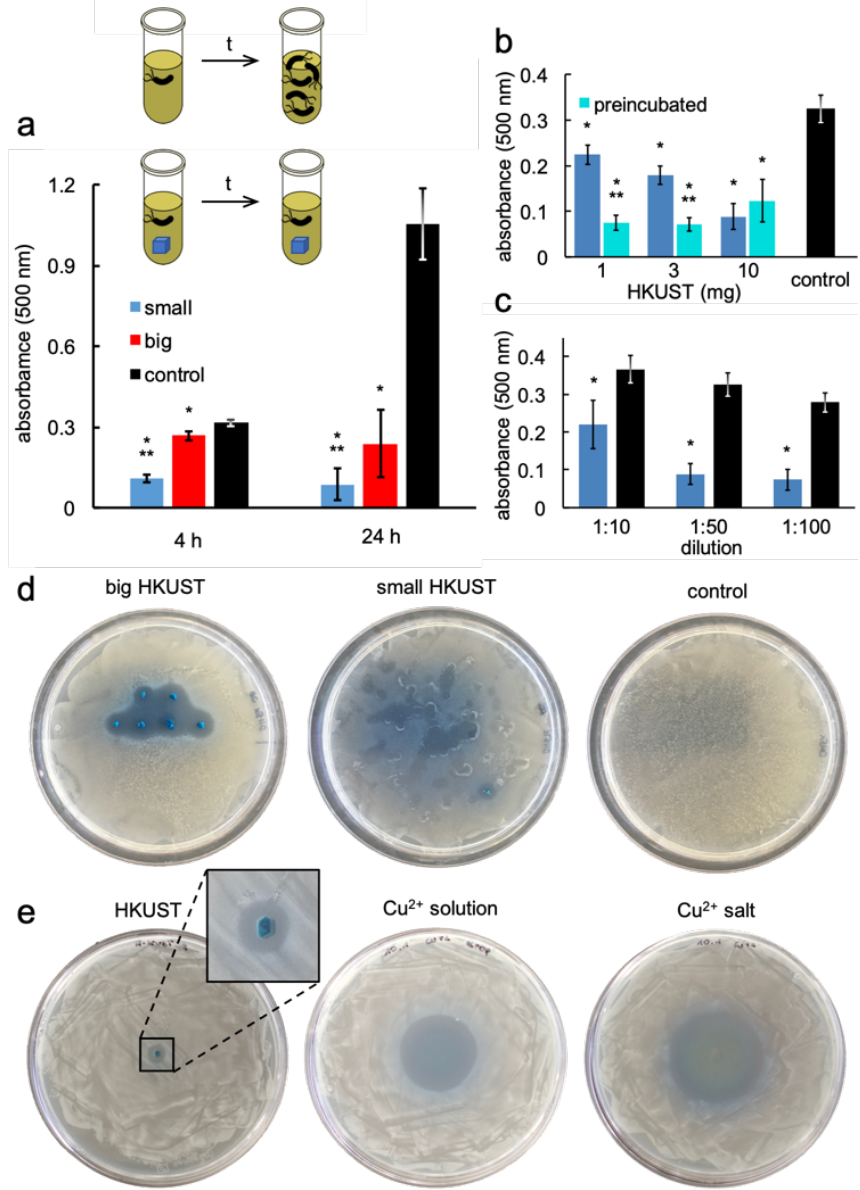

Figure 3: Bacterial population control with macroscopic HKUST crystals a) Absorbance of bacterial cultures (LB) in presence of HKUST (either small or big) at 4 and $24 \mathrm{~h}$. b, c) Absorbance of bacterial cultures growing in exponential phase with and without HKUST by varying the amount of material or the dilution factor. Error bars represent standard deviations over biological replicates ( $n=3$, except $n=6$ for big crystals). *Statistical significance with respect to control. ${ }^{* \star}$ Statistical significance of small vs. big (a, although marginal at $24 \mathrm{~h}$ ) or preincubated vs. non-preincubated (b). d) Petri dishes (LB-agar) seeded with bacteria and set with big HKUST crystals (left), small ones (middle), or without (right). e) Petri dishes seeded with bacteria and set with a big HKUST crystal (left), a solution of $\mathrm{Cu}\left(\mathrm{NO}_{3}\right)_{2}$ (middle), or a powder of $\mathrm{Cu}\left(\mathrm{NO}_{3}\right)_{2}$ (right). Images taken after overnight incubation. 
inhibition was significantly higher with smaller crystals (Welch's $t$-tests, two-tailed $P<0.05$ ), in tune with the differential copper release previously shown (Fig. 2b).

It is important to note that the efficacy of the system depends on the material amount in the medium and the initial bacterial density. To appreciate these issues, we then performed a series of complementary assays with diluted E. coli cultures already in exponential phase (i.e., when bacteria replicate at their maximal speed). We first measured how different amounts of small HKUST-1 crystals $(1,3$, and $10 \mathrm{mg})$ repress bacterial growth (Welch's $t$-tests, two-tailed $P<0.05$ ), revealing a progressive effect (Fig. 3b). We repeated these measurements when crystals had the opportunity to release copper before bacteria appeared (material preincubation), observing now that even $1 \mathrm{mg}$ was enough to keep under control the population (inhibition significantly higher with respect to non-preincubation; Welch's $t$-test, two-tailed $P<0.05)$. Second, we studied how different dilutions $(1: 10,1: 50$, and $1: 100)$ of bacterial cultures impact the system, finding repression in all cases (Welch's $t$-tests, two-tailed $P<0.05$ ) and again a progressive effect (Fig. 3c). Collectively, these data promise to be important to develop accurate quantitative models of real (preclinical) scenarios ${ }^{21}$.

In addition, HKUST- 1 crystals were also able to inhibit bacteria in solid medium (LB-agar; Fig. 3d). For that, we placed big and small HKUST-1 crystals in plates seeded homogenously with bacteria, finding the day after halos around the crystals (for initial plates, see Fig. S7 in SI). Halos even overlapped when particles were close.

Notably, a slow release of antimicrobial agents by autonomous structures is key to achieve a more localized effect. This can allow obtaining higher levels of these agents at a given point minimizing systemic toxicity, which can enhance the effectiveness of a treatment ${ }^{22}$. To illustrate that macroscopic HKUST-1 crystals are suitable elements to achieve this spatiotemporal behavior, we placed in plates seeded homogenously with bacteria, on the one hand, a big crystal $(8.1 \mathrm{mg})$ and, on the other hand, either a microdrop of a $\mathrm{Cu}^{2+}$ solution $(5 \mu \mathrm{L}, 9.7 \mathrm{mg})$ or a microspoon of a $\mathrm{Cu}^{2+}$ salt $(10.1 \mathrm{mg})$, ensuring the same metal amount. Suggestively, we found that the bacterial repression halo produced by the crystal $(\sim 7 \mathrm{~mm}$ diameter) was substantially smaller than the halo produced by the microdrop ( $26 \mathrm{~mm}$ diameter) or the microspoon ( $34 \mathrm{~mm}$ diameter; Fig. 3e).

In addition to copper, HKUST-1 crystals release to the medium benzene-1,3,5-tricarboxylic acid (BTC; Fig. 1), the organic ligand in this MOF ${ }^{15}$. Thus, we decided to explore the corresponding dose-response surface (with separate compounds). From diluted E. coli cultures, we observed that $\mathrm{Cu}^{2+}$ inhibits bacterial growth when its concentration is above $2.5 \mathrm{mM}$ (see Fig. S8 in SI). Following Fig. S4, this concentration is reached after $\sim 16.7 \mathrm{~h}$ of material incubation at $37^{\circ} \mathrm{C}$ without shaking. However, when shaking is applied, a faster profile was observed, reaching that threshold after just $\sim 2.2 \mathrm{~h}$ (see Fig. S9 in SI; likely due to mechanical effects). In this case, we fitted $k=0.2711 \pm 0.0471 \mathrm{~h}^{-1}$ and $x_{\max }=0.1413$ $\pm 0.0089\left(R^{2}=0.9444\right)$. While, for BTC the threshold is at $7.5 \mathrm{mM}$ (Fig. S8). Interestingly, BTC alone can repress bacteria, which agrees with the use of phenolic acids as antiseptic agents ${ }^{23}$. Yet, because the inhibition threshold is 3-fold lower for $\mathrm{Cu}^{2+}$ and there is more metal than ligand molecules in this MOF (3:2 ratio), we can argue that BTC contributes less substantially than $\mathrm{Cu}^{2+}$ to bacterial repression when using the material for this purpose.

Subsequently, we studied to what extent HKUST-1 crystals can be reused. For that, we cultured bacteria in the presence of crystals $(10 \mathrm{mg})$ for long time $(>10 \mathrm{~h})$, showing repression, and then we recycled those crystals to constrain a new bacterial culture. We did so in liquid and solid media, obtaining repression again (see Fig. S10 in SI). Certainly, the macroscopic size of the material was instrumental to achieve such reusability, allowing the manual collection and disposition of individual MOF particles. Our experiments further revealed that $\sim 3 \mathrm{mg}$ material were decomposed into metal and ligand after 24 $\mathrm{h}$ in LB with shaking, leading to a final $\mathrm{Cu}^{2+}$ concentration of $\sim 5.6 \mathrm{mM}$ (Fig. S9). This indeed agrees with our results with preincubated materials. In terms of activity, reused big crystals were shown less efficient (36\% vs. $77 \%$ when fresh; likely due to $30 \%$ mass loss). In addition, $\sim 3.2 \mathrm{mg}$ material were decomposed after $5 \mathrm{~d}$ in LB-agar (note that we observed fully decomposition at very long times), which is a much slower process. Accordingly, consistent activity was observed after different cycles in solid medium (halos of equal diameter; Fig. S10).

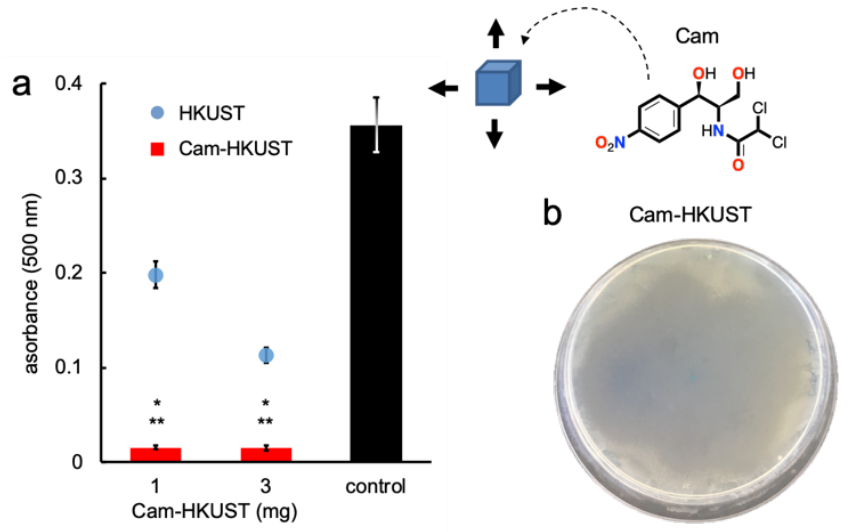

Figure 4: Bacterial population control with macroscopic HKUST crystals loaded with chloramphenicol (Cam-HKUST). a) Absorbance of bacterial cultures (LB) in presence of Cam-HKUST at $4 \mathrm{~h}$. Error bars represent standard deviations over biological replicates $(n=3)$. *Statistical significance with respect to control. ${ }^{* *}$ Statistical significance of the enhancement over mere HKUST crystals. b) Petri dish (LB-agar) seeded with bacteria and set with Cam-HKUST crystals. Image taken after overnight incubation.

Finally, we investigated the ability to exploit macroscopic HKUST-1 crystals as molecular containers. Aiming at maximizing the utility of this material to control bacterial populations, we loaded crystals with a model antibiotic. We considered chloramphenicol, which is a broad-spectrum antibiotic with mainly bacteriostatic mode of action ${ }^{24}$, as well as copper (see Fig. S11 in SI). For that, already prepared HKUST-1 crystals of $\sim 0.2 \mathrm{~mm}$ edge were immersed in a saturated solution of chloramphenicol (see Fig. $\mathbf{S 1 2}$ in $\mathbf{S I}$ ). We quantified in $\sim 7 \%$ (mass) the presence of the guest molecule into the material (through ethanol washes, absorbance at 280 $\mathrm{nm}$ ), meaning that only the pores more in the periphery of the material were occupied.

From diluted $E$. coli cultures in liquid medium (1:100 from overnight), we found significant repression with these new crystals (at $4 \mathrm{~h}$, with 1 and $3 \mathrm{mg}$; Welch's $t$ tests, two-tailed $P<0.05$; Fig. 4a). Even with $1 \mathrm{mg}$ material and without preincubation, the population was tightly controlled. Indeed, $1 \mathrm{mg}$ material leads to a 
maximal concentration of released chloramphenicol of $\sim 35 \mu \mathrm{g} / \mathrm{mL}$ (in $2 \mu \mathrm{mL}$ culture), which is the standard concentration for resistance selection. Thus, a low loading percentage was not an obstacle here. Note also that bacteria grew about 10 times more with mere HKUST-1 crystals (Fig. 4a; Welch's $t$-tests, two-tailed $P<0.05$ ). Consequently, we inferred that guest molecules release much faster than copper (perhaps, $k>0.4 \mathrm{~h}^{-1}$ ) in these MOF particles. We further assessed the contribution of chloramphenicol to the joint antibiotic-MOF action by playing with a concentration gradient, with fixed $3 \mathrm{mg}$ material, pointing out how sublethal chloramphenicol levels (around $1 \mu \mathrm{g} / \mathrm{mL}$ ) can work relatively well in the presence of MOF-derived $\mathrm{Cu}^{2+}$ ions (see Fig. S13 in SI).

Expectedly, chloramphenicol-loaded HKUST-1 crystals were also able to inhibit bacteria in solid medium (Fig. 4b). As before, we placed crystals in plates seeded homogenously with bacteria, but this time we did not observe a set of halos (compare with Fig. 3d, middle). Instead, we found a large region in which bacteria did not grow, suggesting a fast release and spread of the guest molecule (for initial plate, see Fig. S14 in SI).

\section{Conclusion}

In conclusion, this work shows that a given MOF prepared to be observable (size $>0.2 \mathrm{~mm}$ ) can be repurposed as an alternative element for bacterial population control. We found that HKUST-1 crystals are slowly decomposed into their constituents $\left(\mathrm{Cu}^{2+} \text { and } \mathrm{BTC}\right)^{15}$ in biological media, which function as antimicrobial agents (mainly bacteriostatic). Note that this is not the case in pure water. Because of the size of the material, such a decomposition leads to a continuous, but slow release of antimicrobials, even for several days (at $37^{\circ} \mathrm{C}$ we quantified it in $\sim 3 \mathrm{~d}$, without affecting the material-medium equilibrium). In addition, we proved that this material can be loaded with a suitable antibiotic (chloramphenicol, which fits inside the pore) to enhance the antimicrobial action.

This allowed the control of bacterial populations (E. coli) in liquid (LB) or solid (LB-agar) media; a complex spatiotemporal process that depends on the initial bacterial density and the amount of material disclosed. In solid media, the slow release ensured a more localized action. Moreover, thanks to have $\mathrm{mm}$-sized materials, we were able to precisely reuse them in different days against different populations, something that certainly aligns with a sustainable development.

In the literature, there are several game-changing examples of porous nanomaterials (typical size $<200 \mathrm{~nm}$ ) that go towards the miniaturization or substitution of conventional systems for the administration of biochemical compounds 5,6 For example, $\mathrm{Fe}^{3+}$-based MOFs (MIL-53 and variants) ${ }^{25}$. Our results contribute to shift the paradigm, as they rely on MOFs of macroscopic size (jumping 3-4 orders of magnitude) that can be at the same time containers of guest molecules and self-degrading reservoirs of metals. We can imagine several scenarios in which a programmed supply of different bioactive ions/molecules can be highly beneficial. For example, the antibiotic dose might be reduced if metals were at play. They might also be used to enhance the activity of established drugs in preclinical assays ${ }^{26}$. Moreover, different guest molecules might be released according to a predefined temporal pattern if we filled such particles of big size in consecutive steps. From a systems biology perspective ${ }^{27}$, a slow release might be interesting to study the response of biological systems to ramp-like instead of step-like stimuli, trying to unveil overlooked features in stress response $\mathrm{e}^{28}$.
Future work should be focused on testing the ability of macroscopic HKUST-1 crystals (loaded with antibiotic or not) to repress pathogenic bacteria ${ }^{17}$, both in vitro and in vivo (with animal models). However, a limitation of this technology is the fact that BTC is a relatively small ligand, which constrains the palette of guest molecules (at the same time that hinders the loading). Hence, future work should also investigate the possibility of preparing other mm-sized MOFs, with wider pores and based on different metals ${ }^{14}$ (e.g., silver or zinc, which also present antibacterial properties ${ }^{9}$ ). Moreover, as these materials admit multiple post-synthetic modifications (metal exchange, ligand exchange, surface modification, etc. $)^{29}$, there is a great room for maneuver.

\section{Associated content}

Supporting Information (SI) available, containing a description of materials and methods and 13 additional figures.

\section{Authors' information}

Correspondence: * rafael.ballesteros-garrido@uv.es (R.B-G.), * guillermo.rodrigo@csic.es (G.R.).

Contributions: R.B-G. and G.R. conceived this study. R.B-G. and R.M-M. performed the experiments. G.R. analyzed the data and composed the final figures with the help of R.B-G. and R.M-M. G.R. wrote the manuscript. ${ }^{b}$ Equal contribution.

Notes: The authors declare that there are not conflicts of interest.

\section{Acknowledgements}

Work supported by the Spanish Ministry of Economy, Industry, and Competitiveness (CONSOLIDER CTQ2017-90852REDC). Assistance provided by the UV Central Service for Experimental Research and the NANBIOSIS U26 platform.

\section{References}

1 World Health Organization (2018) World health statistics: monitoring health for the sustainable development goals. Geneva.

2 Laxminarayan R, Duse A, Wattal C, Zaidi AK, Wertheim HF, Sumpradit N, Vlieghe E, Hara GL, Gould IM, Goossens H, Greko C, So AD, Bigdeli M, Tomson G, Woodhouse W, Ombaka E, Peralta AQ, Qamar FN, Mir F, Kariuki S, Bhutta ZA, Coates A, Bergstrom R, Wright GD, Brown ED, Cars O (2013) Antibiotic resistance - the need for global solutions. Lancet Infect. Dis. 13, 1057-1098

3 Hawgood S, Hook-Barnard IG, O'Brien TC, Yamamoto KR (2015) Precision medicine: beyond the inflection point. Sci. Transl. Med. 7, 300ps17.

4 Huh AJ, Kwon YJ (2011) Nanoantibiotics: a new paradigm for treating infectious diseases using nanomaterials in the antibiotics resistant era. J. Control. Release 156, 128-145.

5 Aznar E, Oroval M, Pascual L, Murguía JR, Martínez-Máñez R, Sancenón F (2016) Gated materials for on-command release of guest molecules. Chem. Rev. 116, 561-718.

6 Horcajada P, Gref R, Baati T, Allan PK, Maurin G, Couvreur P, Férey G, Morris RE, Serre C (2012) Metal-organic frameworks in biomedicine. Chem. Rev. 112, 1232-1268.

7 Corma A (1997) From microporous to mesoporous molecular sieve materials and their use in catalysis. Chem. Rev. 97, 2373-2420.

8 Rodríguez HS, Hinestroza JP, Ochoa-Puentes C, Sierra CA, Soto CY (2014) Antibacterial activity against Escherichia coli of Cu-BTC (MOF-199) metal-organic framework immobilized onto cellulosic fibers. J. Appl. Polym. Sci. 131, 40815.

9 Demirci S, Ustaoğlu Z, Yılmazer GA, Sahin F, Baç N (2014) 
Antimicrobial properties of zeolite-X and zeolite-A ion-exchanged with silver, copper, and zinc against a broad range of microorganisms. Appl. Biochem. Biotechnol. 172, 1652-1662.

10 Dobrovolskaia MA, Aggarwal P, Hall JB, McNeil SE (2008) Preclinical studies to understand nanoparticle interaction with the immune system and its potential effects on nanoparticle biodistribution. Mol. Pharm. 5, 487-495.

11 Whitesides GM (2003) The right size in nano-biotechnology. Nat. Biotechnol. 21, 1161-1165.

12 Titze T, Lauerer A, Heinke L, Chmelik C, Zimmermann NE, Keil FJ, Ruthven DM, Kärger J (2015) Transport in nanoporous materials including MOFs: The applicability of Fick's laws. Angew. Chem. Int. Ed. 54, 14580-14583.

13 Liu Y, Feng Y, Yao J (2016) Recent advances in the direct fabrication of millimeter-sized hierarchical porous materials. RSC Adv. 6, 80840-80846.

14 Furukawa H, Cordova KE, O'Keeffe M, Yaghi OM (2013) The chemistry and applications of metal-organic frameworks. Science 341, 1230444.

15 Chui SSY, Lo SMF, Charmant JPH, Orpen AG, Williams ID (1999) A chemically functionalizable nanoporous material $\left[\mathrm{Cu}_{3}(\mathrm{TMA})_{2}\left(\mathrm{H}_{2} \mathrm{O}\right)_{3}\right]_{\mathrm{n}}$. Science 283, 1148-1150

16 Li L, Sun F, Jia J, Borjigina T, Zhu G (2013) Growth of large single MOF crystals and effective separation of organic dyes. CrystEngComm 15, 4094-4098.

17 Peleg AY, Hooper DC (2010) Hospital-acquired infections due to gram-negative bacteria. N. Engl. J. Med. 362, 1804-1813.

18 Dupont CL, Grass G, Rensing C (2011) Copper toxicity and the origin of bacterial resistance - new insights and applications. Metallomics 3, 1109-1118.

19 Burtch NC, Jasuja H, Walton KS (2014) Water stability and adsorption in metal-organic frameworks. Chem. Rev. 114 10575-10612.

20 Álvarez JR, Sánchez-González E, Pérez E, SchneiderRevueltas E, Martínez A, Tejeda-Cruz A, Islas-Jácome A, González-Zamora E, Ibarra IA (2017) Structure stability of HKUST-1 towards water and ethanol and their effect on its $\mathrm{CO}_{2}$ capture properties. Dalton Trans. 46, 9192-9200.

21 Nielsen El, Friberg LE (2013) Pharmacokineticpharmacodynamic modeling of antibacterial drugs. Pharmacol. Rev. 65, 1053-1090.

22 Nandi SK, Mukherjee P, Roy S, Kundu B, De DK, Basu D (2009) Local antibiotic delivery systems for the treatment of osteomyelitis - A review. Mater. Sci. Eng. C 29, 2478-2485.

23 Cueva C, Moreno-Arribas MV, Martín-Álvarez PJ, Bills G, Vicente MF, Basilio A, López Rivas C, Requena T, Rodríguez JM, Bartolomé B (2010) Antimicrobial activity of phenolic acids against commensal, probiotic and pathogenic bacteria. Res. Microbiol. 5, 372-382.

24 Malik VS (1972) Chloramphenicol. Adv. Appl. Microbiol. 15, 297-336.

25 Horcajada P, Chalati T, Serre C, Gillet B, Sebrie C, Baati T, Eubank JF, Heurtaux D, Clayette P, Kreuz C, Chang J-S, Hwang YK, Marsaud V, Bories P-N, Cynober L, Gil S, Férey G, Couvreur P, Gref R (2010) Porous metal-organic-framework nanoscale carriers as a potential platform for drug delivery and imaging. Nat. Mater. 9, 172-178.

26 Lun X, Wells JC, Grinshtein N, King JC, Hao X, Dang N-H, Wang X, Aman A, Uehling D, Datti A, Wrana JL, Easaw JC, Luchman A, Weiss S, Cairncross JG, Kaplan DR, Robbins SM, Senger DL (2016) Disulfiram when combined with copper enhances the therapeutic effects of temozolomide for the treatment of glioblastoma. Clin. Cancer Res. 22, 3860-3875.

27 Alon U (2006) An introduction to systems biology: design principles of biological circuits. Chapman and Hall/CRC.

28 Young JW, Locke JC, Elowitz MB (2013) Rate of environmental change determines stress response specificity. Proc. Natl. Acad. Sci. USA 110, 4140-4145.

29 Yin Z, Wan S, Yang J, Kurmoo M, Zeng MH (2017) Recent advances in post-synthetic modification of metal-organic frameworks: New types and tandem reactions. Coord. Chem. Rev. 378, 500-512.

\section{Table of contents graphic}

\section{macroscopic HKUST}

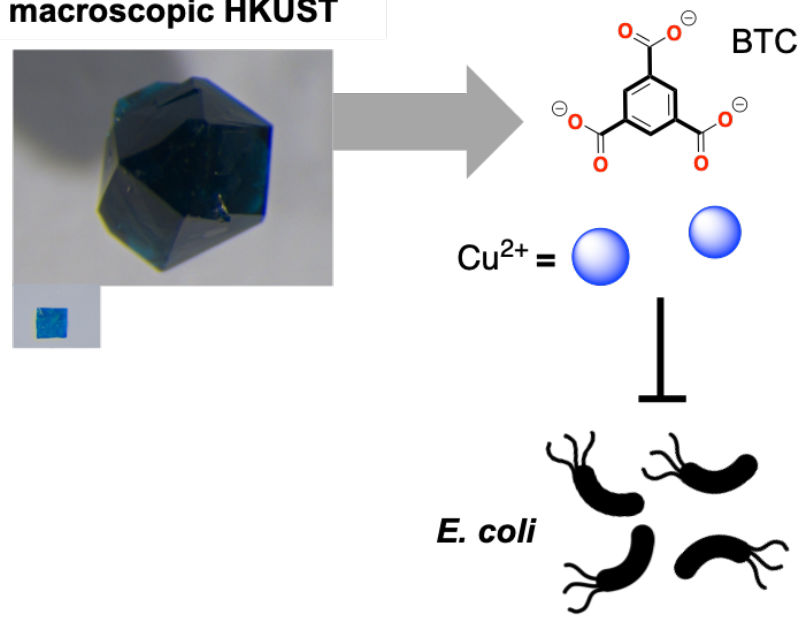

